\title{
GESTÃO DO CONHECIMENTO EM BIBLIOTECAS: UMA REVISÃO SISTEMÁTICA
}

\author{
Michelle Pinheiro \\ mipinheiro78@gmail.com \\ Fernando José Spanhol \\ profspanhol@gmail.com
}

\section{Resumo}

Sendo um assunto de suma importância para as organizações na sociedade atual, a gestão do conhecimento também precisa ser incorporada pelas bibliotecas para melhoria dos processos e serviços acompanhando assim as mudanças, em especial as tecnológicas, e das instituições de ensino das quais fazem parte. O presente artigo visa mapear os estudos científicos dos últimos dez anos acerca da intersecção gestão do conhecimento, bibliotecas e educação. Foi realizada uma revisão sistemática nas bases de dados online Scopus e Web of Science, seguida de uma análise bibliométrica. Os resultados demonstraram que o tema vem crescendo nos últimos anos, e que se relaciona principalmente com a área de Biblioteconomia e Ciência da Informação, como também com as áreas tecnológicas. As palavras-chave que obtiveram maior número de ocorrências foram gestão do conhecimento, bibliotecas acadêmicas, educação, e Biblioteconomia e Ciência da Informação. Constatouse que as bibliotecas já vêm implementando conscientemente modelos, sistemas e práticas de gestão do conhecimento, entretanto no Brasil a gestão do conhecimento em bibliotecas ainda é incipiente.

Palavras-Chave: Gestão do Conhecimento. Bibliotecas. Educação.

\section{INTRODUCC̃̃O}

No atual contexto econômico, a informação e o conhecimento são considerados os principais fatores críticos de sobrevivência organizacional. Tratar esses ativos "[...] de forma articulada e integrada à estratégia organizacional pode se caracterizar como um diferencial de competição global" (ANGELONI; TEIXEIRA; REIS, 2008, p.333).

Segundo Pereira e Bellini (2008), a vantagem competitiva das empresas está em sua capacidade de aprender e no conhecimento acumulado a partir de informações e experiências, o que amplia com o seu uso e gera novos conhecimentos.

Para que uma empresa se torne uma organização de aprendizagem e conhecimento, a mesma precisa entender a importância da Gestão do Conhecimento (GC). Precisa deixar de visualizar o empregado como um mero executor de tarefas, geradores de custos. Precisa valorizar e recompensar seu capital intelectual e fazer com que o conhecimento gerado por ele permaneça na instituição. Necessita criar estratégias que minimize o tempo gasto para execução de tarefas, para que não repita erros cometidos e para que aprimore suas capacidades de converter informação em conhecimento e ações que agreguem valor.

Além de estar presente como um modelo para as organizações, a GC é "uma oportunidade para as bibliotecas renovaremse e reinventarem-se como instituições já bastante abaladas pela crescente disponibilização de conteúdos digitais e pela dificuldade em gerenciá-los" (BEM, 2015, p.30). O conceito surge para as bibliotecas para atender as necessidades da atualidade (LI; LI, 2010), trazendo inovação para seus processos e serviços.

Com base nos aspectos apontados e com o intuito de responder a pergunta de pesquisa: as bibliotecas de instituições de ensino aplicam conscientemente a gestão do conhecimento?, realizou-se uma revisão sistemática da literatura (RSL) em bases de dados online, com posterior análise bibliométrica, objetivando conhecer os estudos científicos acerca da intersecção gestão do conhecimento, bibliotecas e 
educação.

\section{REFERENCIAL TEÓRICO}

Neste capítulo serão abordados inicialmente conceitos relacionados às organizações intensivas do conhecimento, seguido da gestão do conhecimento, e finalizado com a gestão do conhecimento nas bibliotecas.

\subsection{ORGANIZAÇÕES INTENSIVAS EM CONHECIMENTO}

$\mathrm{Na}$ Sociedade do Conhecimento, onde a vantagem competitiva está nas pessoas e nas relações e não mais no capital físico, algumas empresas utilizam o conhecimento como fator inovador, e outras como matéria-prima geradora de bens e serviços. Essas que possuem o conhecimento como base são denominadas de Organizações Intensivas em Conhecimento (OIC) ou Organizações Baseadas no Conhecimento (FREIRE; SPANHOL, 2014).

Segundo Nadai e Calado (2005) OIC refere-se àquelas empresas que utilizam intensamente os ativos intangíveis, tanto para a produção e venda de produtos ou serviços, quanto como resultado de um processo de produção do conhecimento. Para Nonaka, Toyama e Hirata (2008) é considerada uma OIC quando interage dinamicamente com o meio ambiente interno e externo.

Os autores Nadai e Calado (2005) definiram fatores qualitativos que determinam a intensidade do conhecimento na organização bem como a caracteriza ou não como uma OIC, que são: atividades desempenhadas, competências e habilidades das pessoas, produto ou serviço oferecido, mercado de atuação, práticas de GC. Concluem que como o "conhecimento pode ser obtido somente através das pessoas e das ações, experiências, emoções, valores e ideais do indivíduo, é necessário desenvolver práticas contemporâneas de gestão de pessoas e de conhecimento" (NADAI; CALADO, 2005, p.12).

As OICs que prestam serviços, como é o caso das instituições de ensino, podem ser caracterizadas, segundo Antonelli (2000) por: atuarem como fontes primárias de informação e de conhecimento, utilizarem de recursos humanos qualificados comparado a outros setores da economia, gerarem conhecimento útil para a própria organização.

\subsection{GESTÃO DO CONHECIMENTO}

Para Davenport e Prusak (1998, p.6) o conhecimento tem sua origem na informação, e pode ser definido como "[...] uma mistura fluida de experiência condensada, valores, informação contextual e insight experimentado, a qual proporciona uma estrutura para a avaliação e incorporação de novas experiências e informações". O conhecimento de interesse das organizações é aquele que auxiliará na tomada de decisões, na solução de problemas e na criação de novas oportunidades.

De acordo com Takeuchi e Nonaka (2008, p.203) "a criação do conhecimento organizacional, portanto, deve ser entendida como um processo que amplifica 'organizacionalmente' o conhecimento criado por indivíduos e cristaliza-o como parte da rede de conhecimentos da organização". Para que isso ocorra, é necessário que haja atuação e mudança do conhecimento tácito, ou seja, o conhecimento subjetivo, pessoal e difícil de ser transferido.

Para a criação do conhecimento os mesmos autores apresentam duas dimensões: a epistemológica, fundamentada nos conhecimentos tácito e explícito, e a ontológica representada pelo modelo SECI (Socialização, Externalização, Combinação e Internalização). Ainda segundo os autores, a interação entre o conhecimento tácito e $\mathrm{o}$ conhecimento explícito criam quatro modos de conversão do conhecimento, que são:

- Socialização - de tácito para tácito: processo de compartilhamento de experiências, de partilhar seu conhecimento tácito com outro.

- Externalização - tácito para explícito: processo de formalização do conhecimento tácito.

- Combinação - explícito para explícito: processo de compartilhamento de conhecimentos explícitos resultando em novos conhecimentos.

- Internalização - explícito para tácito: processo de incorporação do conhecimento explícito em conhecimento tácito.

Segundo Dalkir (2011, p.4), a GC é a "coordenação deliberada e sistemática das pessoas, da tecnologia, dos processos e da estrutura organizacional de uma organização, a fim de agregar valor através da reutilização e da inovação". Isto é alcançado por meio da 
promoção da criação, do compartilhamento e aplicação dos conhecimentos, assim como do incentivo às melhores práticas para a aprendizagem contínua da organização.

Davenport e Prusak (1998) citam quatro etapas do processo de GC: geração, codificação,

transferência

$\mathrm{ou}$

compartilhamento, e aplicação ou apropriação do conhecimento. Já Probst, Raub e Romhardt (2002) definiram seis processos que servem como pilares para a GC, conforme o Quadro 1.

Quadro 1 - Os pilares da gestão do conhecimento

\begin{tabular}{|l|l|}
\hline PILAR & DESCRIÇÃO \\
\hline $\begin{array}{l}\text { Identificar o } \\
\text { conhecimento }\end{array}$ & Significa analisar e descrever o ambiente da empresa. \\
\hline $\begin{array}{l}\text { Adquirir o } \\
\text { conhecimento }\end{array}$ & $\begin{array}{l}\text { Importar parte do conhecimento de fontes externas, através de } \\
\text { relacionamentos com clientes, fornecedores, concorrentes e } \\
\text { parceiros, suprindo as lacunas de conhecimento. }\end{array}$ \\
\hline $\begin{array}{l}\text { Desenvolver o } \\
\text { conhecimento }\end{array}$ & $\begin{array}{l}\text { Ocorre através de diferentes estágios, começando com a } \\
\text { completa falta de entendimento das relações causais envolvidas } \\
\text { em sua situação até o entendimento total que permite o } \\
\text { controle. Baseia-se no fato de que todos os conhecimentos } \\
\text { passam por um processo evolutivo. }\end{array}$ \\
\hline $\begin{array}{l}\text { Distribuir o } \\
\text { conhecimento }\end{array}$ & $\begin{array}{l}\text { Transferência do conhecimento por meio de trocas pessoais } \\
\text { entre indivíduos. }\end{array}$ \\
\hline $\begin{array}{l}\text { Utilizar o } \\
\text { conhecimento }\end{array}$ & $\begin{array}{l}\text { Assegurar a aplicação do conhecimento para o alcance de } \\
\text { benefícios organizacionais. }\end{array}$ \\
\hline $\begin{array}{l}\text { Reter o } \\
\text { conhecimento }\end{array}$ & Seleção, armazenamento e atualização. \\
\hline $\begin{array}{l}\text { Estabelecer } \\
\text { metas }\end{array}$ & $\begin{array}{l}\text { Estabelecer quais habilidades e conhecimentos devem ser } \\
\text { desenvolvidos e em que niveis. }\end{array}$ \\
\hline $\begin{array}{l}\text { Avaliar o } \\
\text { conhecimento }\end{array}$ & Decidir se as metas de conhecimento foram atingidas. \\
\hline
\end{tabular}

Fonte: Adaptado de Probst, Raub e Romhardt (2002) por Pereira, Silva e Pinto (2016)

Podendo ser entendida como a arte de gerar valor a partir de bens intangíveis da organização, a GC tem a tarefa de indicar o caminho para que as organizações explorem tais bens (SVEIBY, 1998).

\subsection{GESTÃO DO CONHECIMENTO EM BIBLIOTECAS}

Num ambiente comunitário inovador, como das universidades, o conhecimento é 'combustível', e é essencial que esse espaço seja propício ao seu compartilhamento. "Como o tesouro de conhecimento e coração de uma universidade, é imperativo que a biblioteca promova atividades de inovação inerentes ao ensino e à investigação científica através de um ambiente de compartilhamento de conhecimentos" (SHUHUAI; et. al., 2009). A aplicação da GC nesse ambiente "justificase pela importância das bibliotecas no elo da corrente de inovação, da qual as universidades participam ativamente" (CASTRO, 2005, p.20).

De acordo com Lankes (2016), as bibliotecas e os bibliotecários facilitam a criação do conhecimento, e trabalham para tornar as pessoas da comunidade mais inteligentes, e o fazem de quatro modos: fornecem acesso, oferecem capacitação, proporcionam um ambiente seguro, e despertam sua motivação para aprender.

A missão da GC em uma biblioteca possui dois níveis: o primeiro trata da conversão de grandes quantidades de conhecimentos tácitos, com o intuito de transformá-los em explícitos, facilitando o acesso e a utilização em toda a biblioteca; e o segundo refere-se a coletar, preservar e disponibilizar acesso aos registros de conhecimento humano ou recursos da biblioteca, estendendo esse conhecimento a outras pessoas e comunidades. Isso dificilmente ocorre em outras organizações (DONG; 2008).

Segundo Di Domenico, De Bona e Fernández (2003), a GC em bibliotecas traz as seguintes vantagens: o encontro de uma diferenciação estratégica; a capacidade de produzir novo conhecimento mediante a experiência, habilidades e mudanças de atitude dentro da cultura organizacional de uma unidade de informação; melhorar a comunicação entre as áreas; identificar e 
descrever as fontes de conhecimento e ser capaz de transferi-lo eficazmente; simplificar os processos, aumentando a produtividade dos serviços e produtos documentais; maior utilização dos recursos da biblioteca; a possibilidade de criação de um círculo virtuoso entre o aprendizado individual e o da organização; a valorização do trabalho dos profissionais e auxiliares; criar um sentimento de pertencimento nos funcionários da biblioteca; definir sistemas e suportes para a tomada de decisão.

Balagué, Düren e Saarti (2016) afirmam que a GC precisa ser parte integrante da estratégia da biblioteca, que o futuro desse espaço será baseado em GC.

\section{PROCEDIMENTOS METODOLÓGICOS}

A abordagem da pesquisa é de natureza exploratória, que tem por finalidade proporcionar maiores informações sobre determinado assunto, facilitando sua delimitação e formulação de hipóteses. "Constitui um trabalho preliminar ou preparatório para outro tipo de pesquisa" (ANDRADE, 2010, p.112).

Quanto aos procedimentos técnicos, utilizou-se da revisão sistemática da literatura (RSL) em bases de dados online. De acordo com Freire (2013) a RSL é um método de levantamento de dados com revisões rigorosas de publicações acadêmicas para identificar selecionar e avaliar os estudos existentes a respeito do tema.

Com a definição da pergunta de pesquisa: as bibliotecas de instituições de ensino aplicam conscientemente a gestão do conhecimento? definiu-se as bases de dados de consulta online, a Scopus e a Web of Science (WoS), que são bases multidisciplinares e com grande representatividade acadêmica em nível mundial.

A pesquisa foi realizada em outubro de 2016, conforme descrito no Protocolo para Busca Sistemática da Literatura da Biblioteca da Universidade Federal de Santa Catarina UFSC (QUADRO 2).

Quadro 2 - Protocolo para Busca Sistemática da Literatura da Biblioteca da UFSC

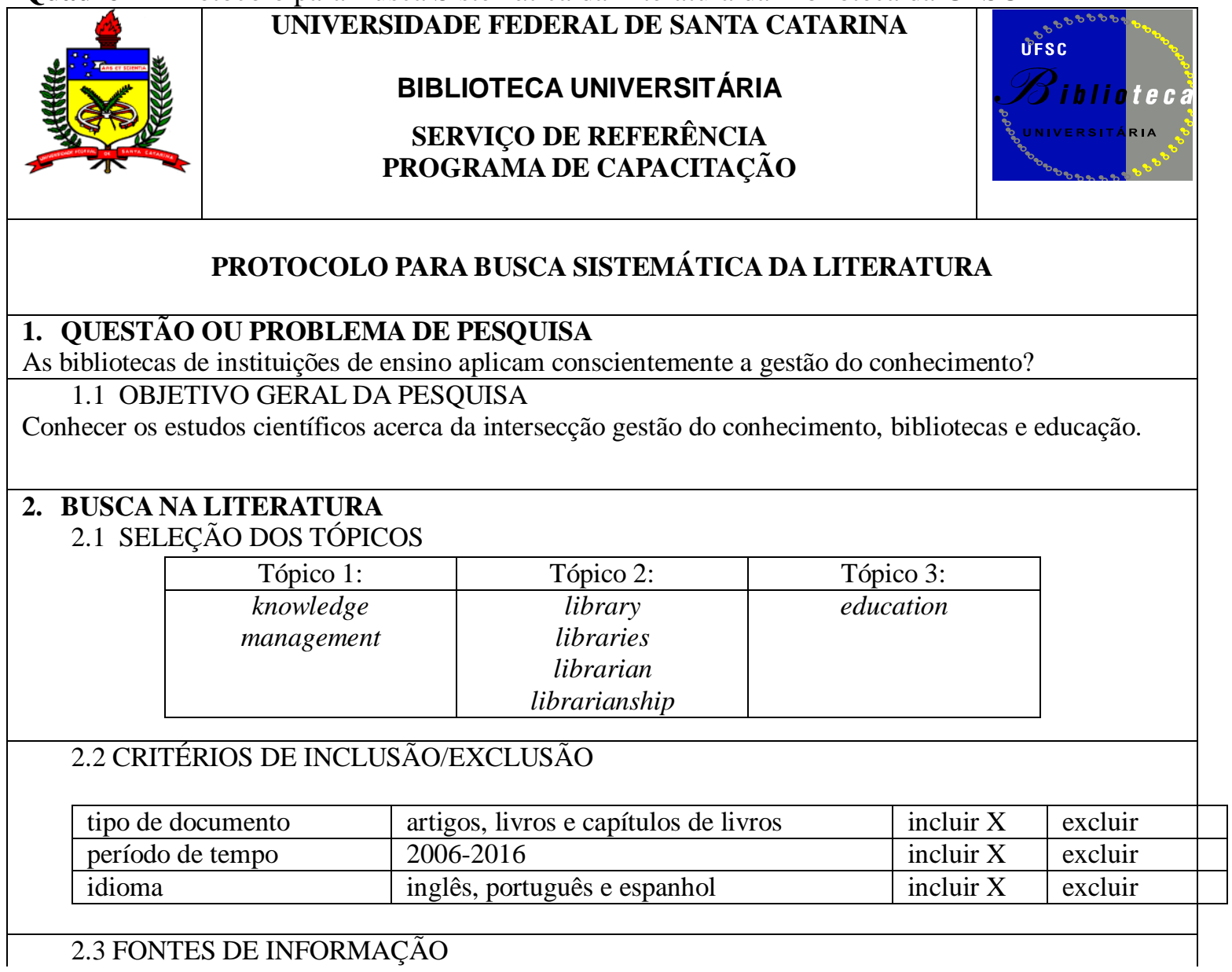




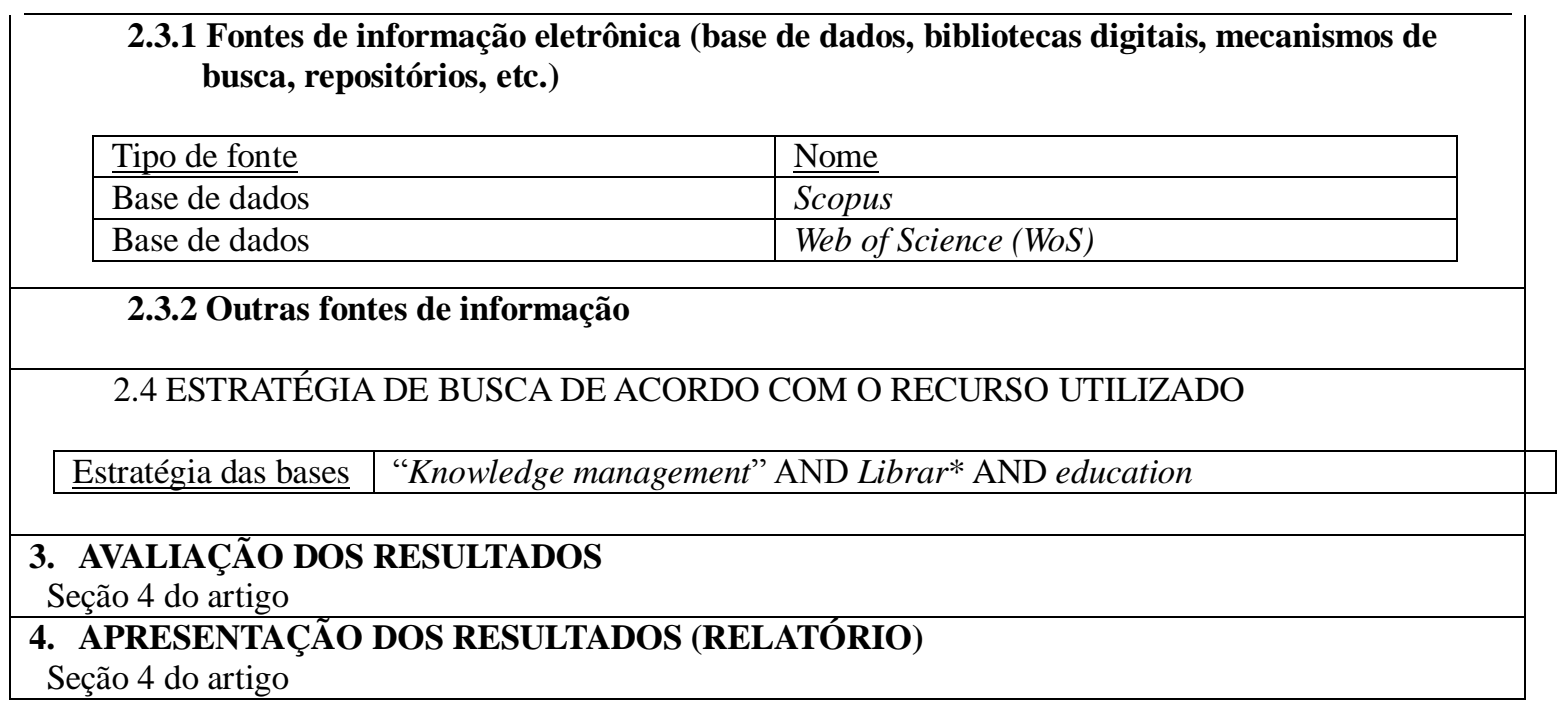

Fonte: Adaptado da BU UFSC pela autora.

Os refinamentos nas bases ocorreram por tipo de documento - artigo, capítulo de livro e livro - e universo temporal, pois o intuito era verificar o que havia nas bases sobre o assunto em questão nos últimos dez anos. A pesquisa resultou num total de 111 documentos, conforme exposto na Tabela 1.
Após a leitura dos títulos, palavras-chave e resumos, foram excluídos 70 artigos pois se repetiam nas bases ou não tinham relação com o escopo da pesquisa, resultando em 41 publicações (TABELA 1).

Tabela 1 - Pesquisa nas bases

\begin{tabular}{lcccc}
\hline TIPO DOC./BASES & SCOPUS & WEB OF SCIENCE & TOTAL & APÓS LEITURA \\
\hline Artigo & 74 & 32 & $\mathbf{1 0 6}$ & $\mathbf{3 6}$ \\
Capítulo de livro & 3 & --- & $\mathbf{3}$ & $\mathbf{3}$ \\
Livro & 2 & -- & $\mathbf{2}$ & $\mathbf{2}$ \\
TOTAL & $\mathbf{7 9}$ & $\mathbf{3 2}$ & $\mathbf{1 1 1}$ & $\mathbf{4 1}$ \\
\hline
\end{tabular}

Fonte: Dados da pesquisa

Prossegue-se então com um tipo de análise resultante de uma RSL, a bibliometria, "um instrumento quantitativo, e com ela é possível que se se minimize a subjetividade que vem junto à recuperação de informações promovendo o conhecimento da área estudada" (ZANINI; PINTO; FILIPPIM, 2013, p.129).

\section{RESULTADOS}

Conforme afirma Freire (2013) existem diferentes métricas que podem ser analisadas pela bibliometria. Em posse dos 41 documentos, buscou-se verificar a evolução e a área do conhecimento das publicações, os países e os autores com maior produção, os tipos de documentos e os mais relevantes, bem como, quais palavras-chave estão presentes.

Considerando a evolução da produção científica nesta temática nos últimos dez anos, observa-se um crescimento a partir de 2011, com picos de produção nos anos de 2013 e 2015, com sete e 12 publicações respectivamente (Gráfico 1). 
Gráfico 1 - Evolução das publicações

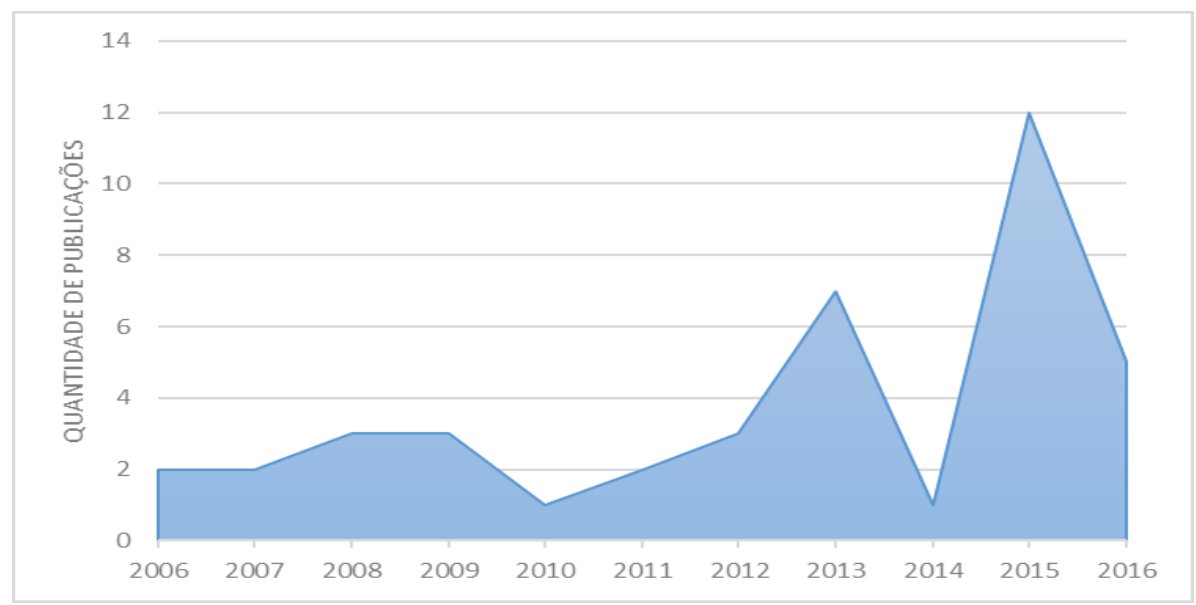

Fonte: Dados da pesquisa

Em contrapartida, em 2014 houve uma queda acentuada com apenas uma publicação. O ano de 2016, com cinco publicações, também foi expressivo, e esse número ainda pode aumentar pois a pesquisa ocorreu no mês de outubro. O total dos últimos seis anos corresponde a quase $61 \%$ da pesquisa.

Com relação as áreas de estudo, elencouse as cinco mais expressivas. Demais áreas foram citadas apenas uma ou duas vezes (Gráfico 2).

Gráfico 2 - Áreas de estudo

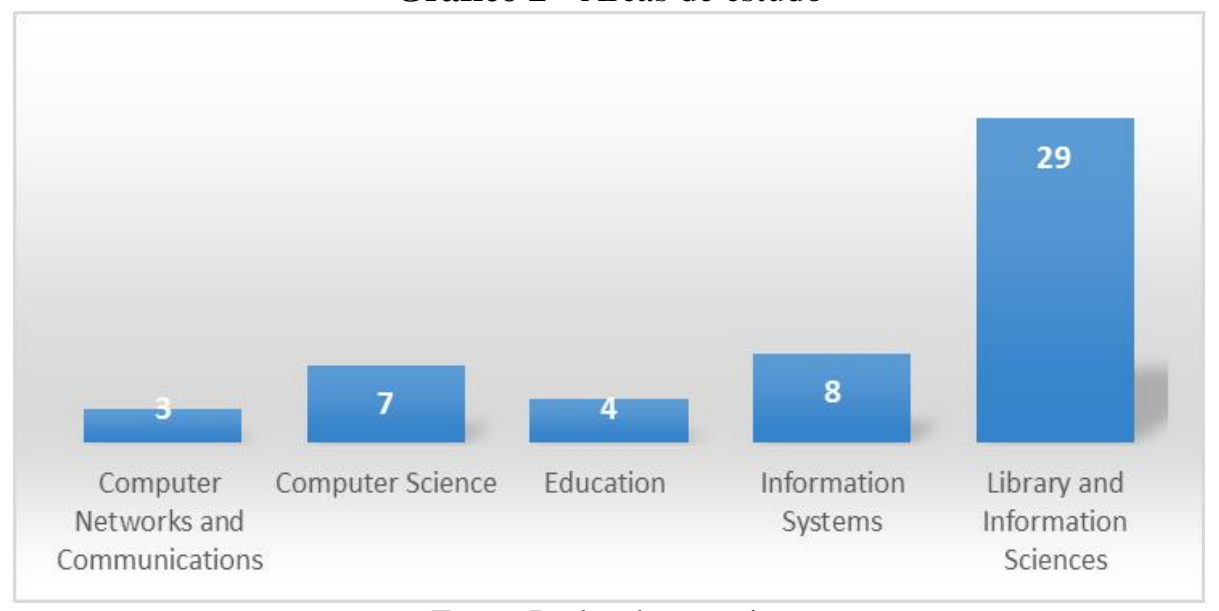

Fonte: Dados da pesquisa

A gestão do conhecimento é considerada por Rokknuzzaman e Umemoto (2009) um assunto de natureza multidisciplinar e de perspectivas variadas. Na temática do estudo a área que obteve grande destaque foi a Biblioteconomia e Ciência da Informação, citada em 29 publicações. Na sequência aparecem as áreas de Sistemas de Informação,
Ciência da Computação, Educação e Redes de Computadores e Comunicações. Nota-se que o tema também tem sido bastante abordado pelas áreas tecnológicas.

Pela análise geográfica, o Gráfico 3 apresenta os países que contribuíram com a temática. 
Gráfico 3 - Países das publicações

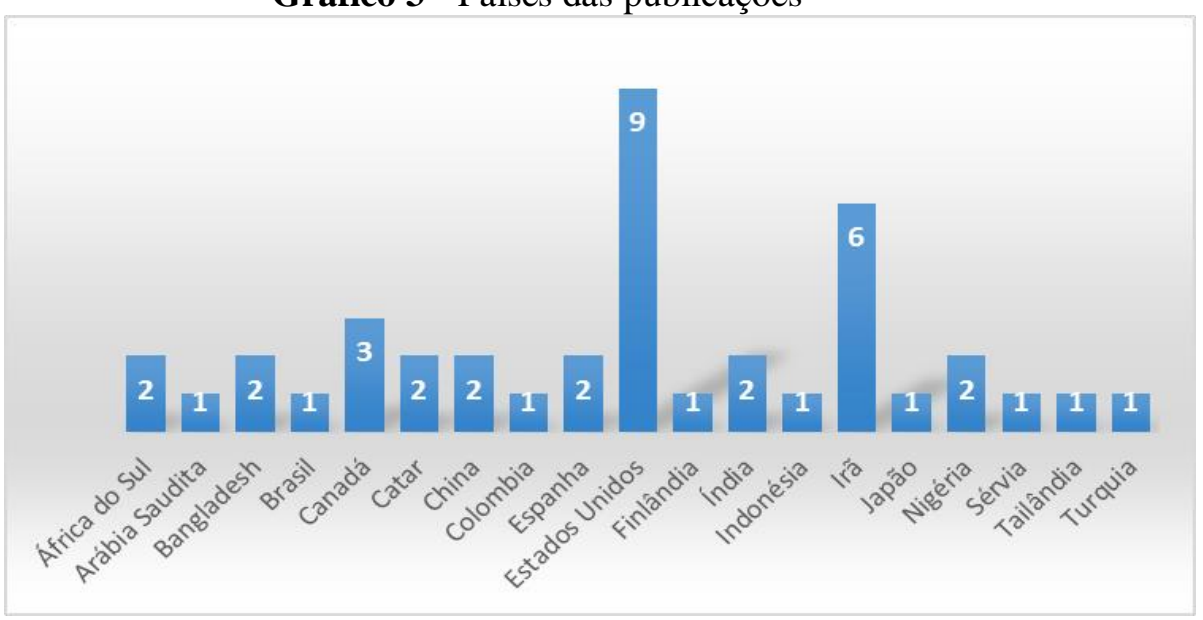

Fonte: Dados da pesquisa

O país com maior número de publicações é o Estados Unidos com nove documentos, seguido do Irã com seis e do Canadá com três, concentrando quase $44 \%$ das publicações. Os demais apresentaram somente um ou dois documentos, como foi o caso do Brasil com apenas $\mathrm{o}$ artigo Knowledge management framework to the university libraries, de Bem, Coelho e Dandolini (2016).

O Gráfico 4 demonstra o quantitativo de publicações por autores, considerando àqueles com duas ou mais publicações.

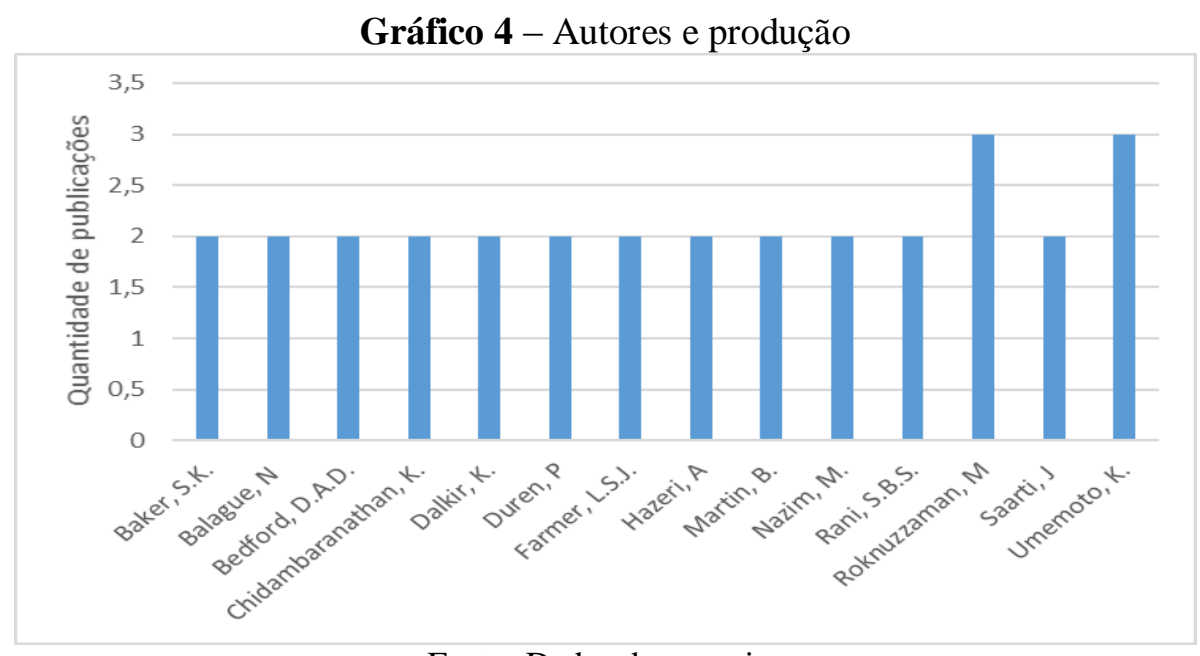

Fonte: Dados da pesquisa

Os autores com maior produção foram Md. Roknuzzaman e Katsuhiro Umemoto, ambos com três artigos em coautoria, dois de 2013 e um de 2008. Entre eles, o mais citado foi o de 2008, com seis citações: Knowledge management's relevance to library and information science: an interdisciplinary approach. Demais autores apresentaram duas publicações cada.

Delimitou-se os tipos de documentos: artigo, capítulo de livro e livro, devido à avaliação prévia, por pares e pelo conteúdo aprofundado (Gráfico 5). 
Gráfico 5 - Tipo de documento

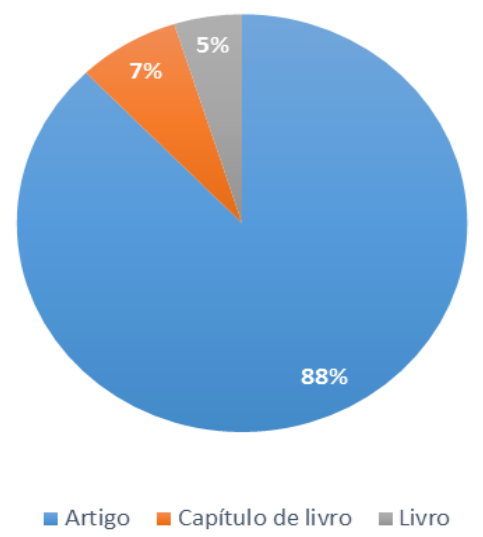

Fonte: Dados da pesquisa

Este Gráfico 5 ilustra a predominância do tipo 'artigo' com 36 itens, correspondendo a $88 \%$ do total, seguido do 'capítulo de livro' com três itens ou 7\%, e do 'livro' com dois itens ou 5\%.

As palavras-chave resultantes da pesquisa, um total de 134 palavras diferentes e indexadas por seus autores, foram copiadas para a ferramenta Wordle $e^{l}$ onde formou-se a "nuvem de palavras". A ferramenta permite a visualização das palavras em tamanho proporcional a frequência em que as mesmas aparecem no texto fonte, bem como ajustá-las em cores e layouts diferenciados (Figura 1).

Figura 1 - Nuvem de palavras-chave

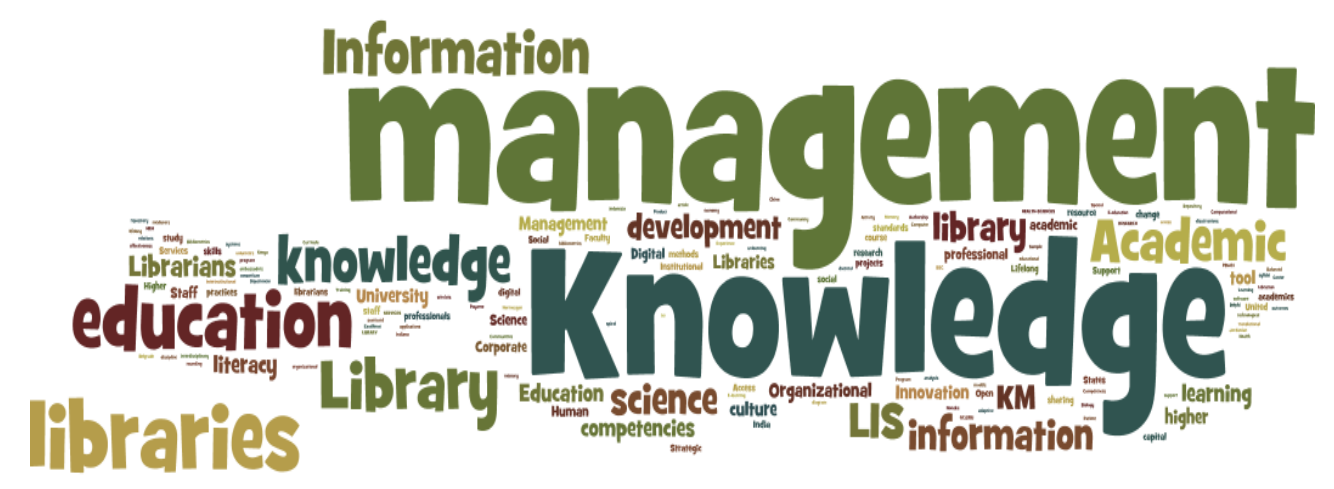

Elaborado pela autora

${ }^{1}$ Disponível gratuitamente em: http://www.wordle.net/. Acesso em: 6 de novembro de 2016. 
Observa-se que as quatro palavras-chave com maior número de ocorrências, por ordem da mais citada para a menos citada, foram knowledge management, academic libraries, education, e library and information science, sendo que a primeira e a terceira foram utilizadas igualmente na pesquisa.

As cinco publicações com maior número de citações estão listadas na Tabela 2, em ordem decrescente:

Tabela 2 - Publicações mais citadas

\begin{tabular}{|c|c|c|c|c|c|c|}
\hline Autor(es) & Título & Ano & $\begin{array}{l}\text { Tipo } \\
\text { Doc. }\end{array}$ & Citações & País & Base \\
\hline $\begin{array}{l}\text { Zong, Q.-J., Shen, } \\
\text { H.-Z., Yuan, Q.-J., } \\
\text { Hu, X.-W., Hou, } \\
\text { Z.-P., Deng, S.-G. }\end{array}$ & $\begin{array}{l}\text { Doctoral dissertations of Library } \\
\text { and Information Science in } \\
\text { China: A co-word analysis }\end{array}$ & 2013 & Artigo & 24 & China & $\begin{array}{l}\text { Scopus e } \\
\text { WoS }\end{array}$ \\
\hline $\begin{array}{l}\text { Davis, H.L., } \\
\text { Somerville, M.M. }\end{array}$ & $\begin{array}{l}\text { Learning our way to change: } \\
\text { Improved institutional alignment }\end{array}$ & 2006 & Artigo & 18 & EUA & Scopus \\
\hline $\begin{array}{l}\text { Tanloet, P., } \\
\text { Tuamsuk, K. }\end{array}$ & $\begin{array}{l}\text { Core competencies for } \\
\text { information professionals of Thai } \\
\text { academic libraries in the next } \\
\text { decade (A.D. 2010-2019) }\end{array}$ & 2011 & Artigo & 11 & Tailândia & Scopus \\
\hline $\begin{array}{c}\text { Shuhuai, R., } \\
\text { Xingjun, S., } \\
\text { Haiqing, L., Jialin, } \\
\text { C. }\end{array}$ & $\begin{array}{l}\text { From information commons to } \\
\text { knowledge commons: Building a } \\
\text { collaborative knowledge sharing } \\
\text { environment for innovative } \\
\text { communities }\end{array}$ & 2009 & Artigo & 10 & China & $\begin{array}{l}\text { Scopus e } \\
\text { WoS }\end{array}$ \\
\hline $\begin{array}{l}\text { Lyon, JA; Tennant, } \\
\text { MR; Messner, KR; } \\
\text { Osterbur, DL }\end{array}$ & $\begin{array}{l}\text { Carving a niche: establishing } \\
\text { bioinformatics collaborations }\end{array}$ & 2006 & Artigo & 9 & EUA & WoS \\
\hline
\end{tabular}

Fonte: Dados da pesquisa

Todos os artigos elencados na Tabela 2 estão disponíveis gratuitamente em texto completo, o que influencia no número de citações. Dos cinco artigos, quatro aparecem na base Scopus e três na WoS, sendo que dois deles aparecem em ambas. Com a leitura dos resumos verificou-se que o documento mais relevante para a pesquisa é o From information commons to knowledge commons: building a collaborative knowledge sharing environment for innovative communities de Shuhuai, et. al. (2009), presente também entre os dez trabalhos analisados no estudo de Bem e Coelho
(2014). Aprofundando a leitura da publicação verificou-se que Shuhuai, et. al. (2009) propõem um novo modelo conceitual, o Knowledge Commons (Figura 2), para apoiar atividades comunitárias inovadoras da biblioteca universitária, visando um ambiente colaborativo de compartilhamento do conhecimento. $\mathrm{O}$ modelo baseia-se em Information Commons, nas teorias de gestão do conhecimento, colaboração e Biblioteca 2.0, visando a integração da biblioteca, dos recursos físicos, virtuais e humanos em um todo. 
Figura 2 - Modelo conceitual para Knowledge Commons (KC)

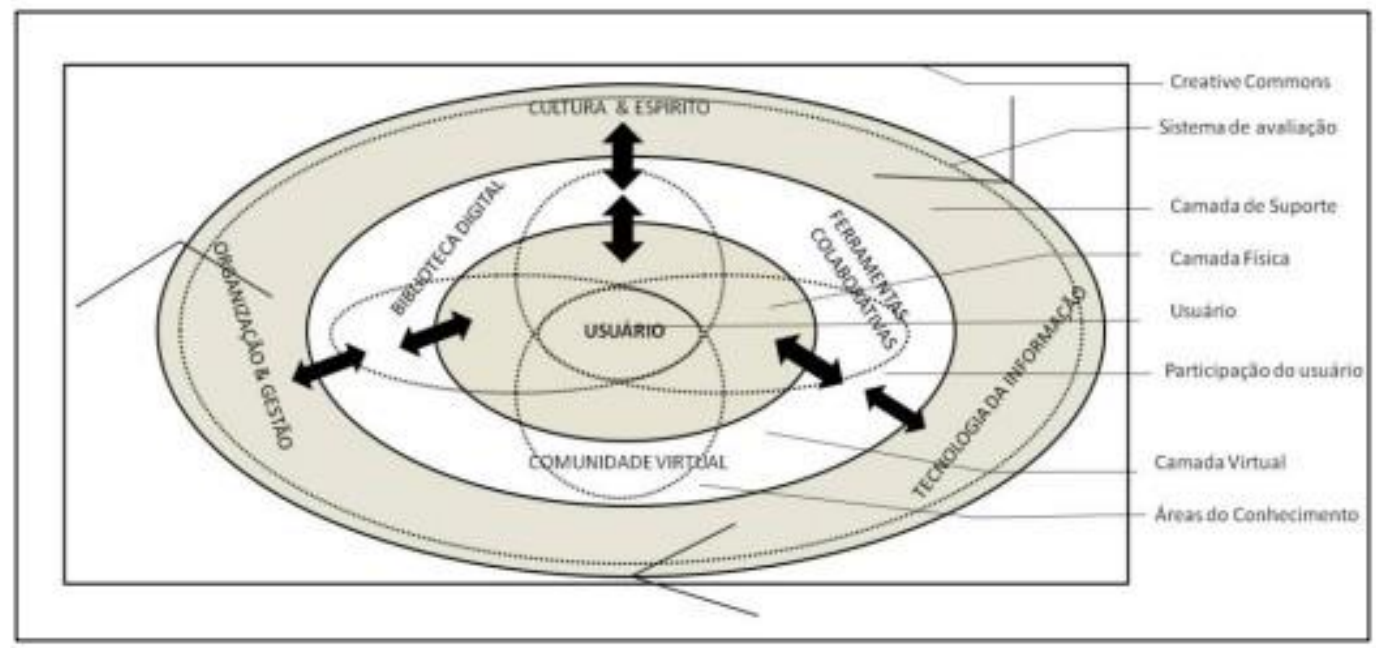

Fonte: Adaptado de Shuhuai, et. al. (2009) por Bem e Coelho (2014, p.160)

As práticas de Information Commons e Biblioteca 2.0 têm desencadeado movimentos nos serviços das bibliotecas, enquanto que o Knowledge Commons seria uma diretriz para um ambiente de compartilhamento de conhecimento tanto para os usuários quanto para a equipe da biblioteca. Estes estarão envolvidos no processo de inovação e as várias camadas do $\mathrm{KC}$ se tornam mais importantes.

Optou-se também por analisar a publicação mais recente que estivesse disponível em texto completo. Chegou-se então ao artigo de 2016 publicado na Finlândia, recuperado pelas duas bases de dados: Comparing the knowledge management practices in selected European higher education libraries de Núria Balagué, Petra Düren, Jarmo Saarti. O objetivo do estudo qualitativo foi investigar a implementação de um sistema de gestão do conhecimento em três diferentes bibliotecas de Europa: IZUS / Universitätsbibliothek Stuttgart, Alemanha; Universitat Autònoma de Barcelona (UAB) Barcelona, Espanha; Universidade da Finlândia Oriental (UEF), Finlândia, fazendo uma comparação dos processos de GC que estão sendo implementados em cada uma, para aprendizagem do pessoal das bibliotecas. O intuito dos autores foi identificar as melhores práticas de cada uma das bibliotecas participantes, para que outras se beneficiassem das experiências. Verificou-se que a tecnologia é um elemento essencial na implementação de GC pois permite uma gestão rigorosa e fácil, entretanto deve ser acompanhada de uma cultura de compartilhamento e valorização do conhecimento. "A implementação de uma ferramenta de GC não é nem dispendiosa nem difícil, mas somente terá sucesso quando a equipe da biblioteca estiver motivada $\mathrm{e}$ disposta a compartilhar seus conhecimentos" (BALAGUÉ; DÜREN; SAARTI, 2016, p.192). Com base no estudo, concluem que a implementação em todas as bibliotecas levaram a uma melhor gestão e inclusão da equipe no desenvolvimento dos serviços.

\section{CONSIDERAÇÕES FINAIS}

Esta pesquisa objetivou conhecer a evolução dos estudos científicos acerca da relação gestão do conhecimento e bibliotecas de instituições de ensino para seu aprimoramento teórico. Para isso utilizou-se da revisão sistemática em bases de dados, seguida da técnica bibliométrica.

Nota-se que a temática está presente com expressividade na área da Biblioteconomia e Ciência da Informação, e também aparece nas áreas tecnológicas. A diversidade de palavraschave, 134 palavras, com destaque para gestão do conhecimento, bibliotecas acadêmicas, educação e biblioteconomia e ciência da informação. Os países com mais publicações foram Estados Unidos, Irã e Canadá. O auge da produção ocorreu nos anos de 2013 e 2015, e tem sido expressivo em 2016, entretanto houve uma queda acentuada em 2014. Ocorreu uma predominância quase que absoluta do tipo de documento 'artigo'. 
Os autores com maior produção foram Md. Roknuzzaman e Katsuhiro Umemoto, ambos com três artigos em coautoria.

Dessa forma alcançou-se o objetivo do estudo, pois constatou-se tanto com a leitura dos resumos quando dos artigos selecionados que as bibliotecas estão caminhando para incorporar a GC no seu dia a dia, como observou-se nos dois artigos, um focando no modelo conceitual para bibliotecas universitárias e outro na implementação de um sistema de gestão do conhecimento nas bibliotecas da Europa. Entretanto é necessário ampliar os estudos a respeito da GC nas bibliotecas, principalmente no Brasil, que nesta pesquisa apareceu apenas um artigo.

\title{
KNOWLEDGE MANAGEMENT IN LIBRARIES: A BIBLIOMETRIC STUDY
}

\begin{abstract}
As a matter of paramount importance for organizations in today's society, knowledge management also needs to be incorporated by libraries to improve processes and services, thus following the changes, especially technological ones, and of the educational institutions of which they are part. The present article aims to map the scientific studies of the last ten years about the intersection knowledge management, libraries and education. A systematic review was performed on the online databases Scopus and Web of Science, followed by a bibliometric analysis. The results demonstrated that the theme has been growing in recent years, and that it is mainly related to the area of Librarianship and Information Science, as well as to the technological areas. The keywords that obtained the highest number of occurrences were knowledge management, academic libraries, education, and Librarianship and Information Science. It was found that libraries have been consciously implementing models, systems and practices of knowledge management; however, in Brazil the management of knowledge in libraries is still incipient.
\end{abstract}

Keywords: Knowledge management. Libraries. Education.

\section{REFERÊNCIAS}

ANDRADE, Maria Margarida de. Introdução à metodologia do trabalho científico: elaboração de trabalhos na graduação. 10.ed. São Paulo: Atlas, 2010.

ANGELONI, Maria Terezinha; TEIXEIRA, Fábio Leandro Moratti; REIS, Eduardo Sguario dos. Portal do conhecimento: integrando estratégias, pessoas e informações. In: ANGELONI, Maria Terezinha (org.). Organizações do conhecimento: infraestrutura, pessoas e tecnologia. 2.ed. rev. e ampl. São Paulo: Saraiva, 2008. cap. 14. p.333-363.

ANTONELLI, C. New information technology and localized technological change in the knowledge-based economy. In: Boden, M.; Miles, I. (Org.). Services and knowledge-based economy. London: Continuum, 2000.
BALAGUÉ, N.; DÜREN, Petra; SAARTI, Jarmo. Comparing the knowledge management practices in selected European higher education libraries. Library Management, v. 37 , n. $4 / 5$ p. $182-194$, 2016. Disponível em:

http://www.emeraldinsight.com/doi/abs/10.11 08/LM-12-2015-0068 . Acesso em: 20 out. 2016.

BEM, Roberta Moraes de. Framework de gestão do conhecimento para bibliotecas universitárias. 2015. 344f. Tese (Doutorado em Engenharia e Gestão do Conhecimento) Universidade Federal de Santa Catarina, Florianópolis, 2015.

BEM, Roberta Moraes de; COELHO, Christianne Coelho de Souza Reinisch. Metodologias, modelos conceituais e frameworks: uma análise da implementação da gestão do conhecimento em bibliotecas. International Journal of 
Knowledge Engineering and Management (IJKEM), v. 3, n. 5, p. 144-173, 2014.

Disponível em:

$<$ http://incubadora.periodicos.ufsc.br/index.p hp/IJKEM/article/viewFile/2460/3253> .

Acesso em: 21 out. 2016.

BEM, Roberta Moraes de; COELHO,

Christianne Coelho de Souza Reinisch;

DANDOLINI, Gertrudes Aparecida.

Knowledge Management Framework to the University Libraries. Library Management, v. 37, n. 4/5, 2016.

CASTRO, G. de. Gestão do conhecimento em bibliotecas universitárias: um instrumento de diagnóstico. 2005. 160 p. Dissertação (Mestrado em Ciência da Informação) - Universidade Federal de Santa Catarina, Florianópolis, 2005. Disponível em: $<$ http://pgcin.paginas.ufsc.br/files/2010/10/C ASTRO-Gardenia.pdf> . Acesso em: 15 set. 2016.

DALKIR, Z. Knowledge management in theory and practice. 2.nd. MIT Press: Cambridge, 2011.

DAVENPORT, Thomas H.; PRUSAK, L. Conhecimento empresarial: como as organizações gerenciam o seu capital intelectual. Rio de Janeiro: Campus, 1998.

DI DOMENICO, Adriana; DE BONA, Graciela Susana; FERNÁNDEZ, Oscar Alberto. La inteligencia en acción: gestionar por el conocimiento. Biblios: Revista Electrónica de Ciencias de la Información, n. 15, 2003. Disponível em: < http://eprints.rclis.org/5542/> . Acesso em: 18 nov. 2016.

DONG, E. X. Using blogs for knowledge management in libraries. CALA Ocasional Paper

Series, n. 2, p. 1-7, nov. 2008.

FREIRE, Patrícia de Sá. Aumente a qualidade e quantidade de suas publicações científicas: manual para elaboração de projetos e artigos científicos. Curitiba: CRV, 2013.

FREIRE, Patrícia de Sá; SPANHOL, Fernando José. O conhecimento organizacional: produto ou

processo?. Perspectivas em Gestão \&

Conhecimento, v. 4, n. 1, p. 3-21, 2014.

Disponível em: <

https://dialnet.unirioja.es/servlet/articulo?codi go $=4800997>$.Acesso em: 20 maio 2016 .

LANKES, R. David. Expect more: melhores bibliotecas para um mundo complexo. São Paulo: FEBAB, 2016.

LI, N.; LI, Q. Knowledge management modes and strategies for university libraries. In: INTERNATIONAL CONFERENCE ON FUTURE INFORMATION TECHNOLOGY AND MANAGEMENT ENGINEERING, 2., 2010, Changzhou. [Conference

Publications...] 2010, p. 288-290.

NADAI, F. C., CALADO, L. R. O conhecimento como recurso estratégico: caracterizando uma organização intensiva em conhecimento (OIC), [2005]. Disponível em: $<$ https://www.researchgate.net/publication/23 7354608_O_conhecimento_como_recurso_es trategico_caracterizando_uma_organizacao_i ntensiva_em_conhecimento_OIC>. Acesso em: 16 nov. 2016.

NONAKA, I; TOYAMA, R.; HIRATA, T. Managing flow: a process theory of the knowledge-based firm. New York: Palgrave MacMillan, 2008.

PEREIRA, Maria Olívia Ferreira; SILVA, Helena de Fátima Nunes; PINTO, José Simão de Paula. A Memória organizacional nos processos de gestão do conhecimento: um estudo na Universidade Federal do Paraná. Informação \& Informação, v. 21, n. 1, p. 348-374, 2016. Disponível em:

<http://www.uel.br/revistas/uel/index.php/inf ormacao/article/view/18253> . Acesso em: 11 nov. 2016

PEREIRA, Rita de Cássia de Faria; BELLINI, Carlo Gabriel Porto. As redes como tecnologia de apoio à gestão do conhecimento. In: ANGELONI, Maria Terezinha (org.).

Organizações do conhecimento: infraestrutura, pessoas e tecnologia. 2.ed. rev. e ampl. São Paulo: Saraiva, 2008. cap. 10. p.245-272.

ROKNUZZAMAN, M. D.; UMEMOTO, K. 
How library practitioners view knowledge management in libraries: a qualitative study. Library Management, v.30, n.8/9, p.643-656, 2009. Disponível em: <www.emeraldinsight.com/0143-5124.htm> . Acesso em: 10 nov. 2016.

ROKNUZZAMAN, M. D.; UMEMOTO, K. Knowledge management's relevance to library and information science: an interdisciplinary approach. Journal of Information \& Knowledge Management, v. 7, n. 04, p. 279-290, 2008.

PROBST, Gilbert; RAUB, Steffen; ROMHARDT, Kai. Gestão do conhecimento: os elementos construtivos do sucesso. Porto Alegre: Bookman, 2002.

SHUHUAI, R. et al. From information commons to knowledge commons: building a collaborative knowledge sharing environment for innovative communities. The Electronic Library, v. 27, n. 2, p. 247-257, 2009.

SVEIBY, Karl Erik. A nova riqueza das organizações: gerenciando e avaliando patrimônios de conhecimento. Rio de Janeiro: Campus, 1998.

TAKEUCHI, H.; NONAKA, I. Gestão do conhecimento. Porto Alegre: Bookman, 2008.

ZANINI, Georgia Barreto; PINTO, Marli Dias Souza; FILIPPIM, Eliane Salete. Análise bibliométrica aplicada à gestão do conhecimento. Conhecimento Interativo, v. 6, n. 2, p. 124-140, 2013. Disponível em: $<$ http://app.fiepr.org.br/revistacientifica/index. $\mathrm{php/conhecimentointerativo/article/view/85>.}$ Acesso em: 8 nov. 2016. 